\title{
Formación inicial docente en Educación Especial en las actividades de acercamiento a la práctica escolar
}

Initial teacher training in Special Education in activities to approach school practice

Formação inicial de professores em Educação Especial em atividades

para abordar a prática escolar

\author{
MAURICIO ZACARÍAS GUTIÉRREZ ${ }^{1}$ \\ MANUEL GREGORIO ORTIZ HUERTA ${ }^{2}$
}

\begin{abstract}
RESUMEN
El artículo analiza la formación inicial docente de los estudiantes de la licenciatura en Educación Especial a partir de las Actividades de Acercamiento a la Práctica Escolar que realizan en las Unidades de Servicio de Apoyo a la Educación Regular (USAER) y en los Centros de Atención Múltiple (CAM). Para ello, desde el enfoque de la investigación cualitativa y ocupando el método de análisis del contenido, se estudiaron los diarios de trabajo que realizan los estudiantes en las Jornadas de Observación y Práctica Docente. Los resultados dan cuenta del saber docente que el estudiante adquiere de las impresiones y vivencias en el centro escolar, como de la práctica educativa que el profesor titular realiza para la enseñanza de los contenidos académicos.
\end{abstract}

Palabras clave: Escuela; formación; saber; práctica docente.

\begin{abstract}
The article analyzes the initial teacher training of the students of the degree in Special Education from the Activities of Approach to the School Practice that they carry out in the Units of Service of Support to the Regular Education (USAER) and in the Centers of Multiple Attention (CAM). For this, from the approach of qualitative research and using the method of content analysis, the work diaries that students make in the Observation and Teaching Practice Days were studied. The results show the teacher's knowledge that the student acquires from the impressions and experiences in the school, as well as the educational practice that the professor carries out for the teaching of academic content.
\end{abstract}

Keywords: School; training; to know; teaching practice.

1. ESCUELA NORMAL DE LICENCIATURA EN EDUCACIÓN PRIMARIA "FRAY MATÍAS DE CÓRDOVA", México | mazag50@gmail.com https://orcid.org/0000-0003-4564-5673

2. ESCUELA NORMAL DE LICENCIATURA EN EDUCACIÓN PRIMARIA “FRAY MATÍAS DE CÓRDOVA”, México | manuelhuerta31101982@gmail.com https://orcid.org/0000-0003-3977-0953 


\section{RESUMO}

O artigo analisa a formação inicial de professores dos alunos da graduação em Educação Especial a partir das Atividades de Abordagem à Prática Escolar que realizam nas Unidades de Serviço de Apoio à Educação Regular (USAER) e nos Centros de Atenção Múltipla (CAM). Para isso, a partir da abordagem da pesquisa qualitativa e utilizando o método de análise de conteúdo, foram estudados os diários de trabalho que os alunos fazem nas Jornadas de Observação e Prática de Ensino. Os resultados mostram o conhecimento do professor que o aluno adquire a partir das impressões e experiências na escola, bem como a prática educacional que o professor realiza para o ensino do conteúdo acadêmico.

Palavras-chave: Escola; treinamento; conhecer; prática de ensino.

\section{INTRODUCCIÓN}

El Sistema Educativo Mexicano actualmente atiende la educación básica, organizada en: preescolar, primaria y secundaria. El plan de estudio para la educación básica que se oferta a la niñez en territorio mexicano es homogénea, así mismo, a los estudiantes que presentan barreras para el aprendizaje se les oferta el mismo plan de estudio, con profesores especializados para atender el servicio de la educación especial.

En atención a los estudiantes que presentan barreras para el aprendizaje, la licenciatura en Educación Especial en México tuvo su última actualización de planes y programas de estudio en el año 2004 -aún vigente- establece que el estudiante tendrá en el trayecto formativo - que dura ocho semestres - tres áreas de actividad: actividades principalmente escolarizadas, de acercamiento a la práctica escolar, y práctica intensiva en condiciones reales de trabajo. Las dos primeras se atienden de primero a sexto semestre y la última en séptimo y octavo.

Asimismo, prescribe cuatro áreas de formación: auditiva y de lenguaje, intelectual, motriz y visual. Sin embargo, en la Escuela Normal Fray Matías de Córdova, solo se forma a los estudiantes en dos de ellas: auditiva y de lenguaje e intelectual. La política interna en la entidad chiapaneca cuando se establece la licenciatura así lo conformó, y que hasta el día de hoy se mantiene.

Si bien el estudiante solo se forma en una de las dos áreas de atención, fortalece el conocimiento teórico a partir de su participación en las escuelas de educación regular de educación básica (preescolar, primaria y secundaria) que cuentan con las Unidades de Servicio de Apoyo a la Educación Regular (Usaer), y en los Centros de Atención Múltiple (CAM). El periodo de tiempo en que se acercan a la Usaer o CAM, está en función de la organización administrativa y de la política interna de la Escuela Normal.

Específicamente el plan de estudio señala seis asignaturas que acercan al estudiante a la práctica escolar. Inicia con Escuela y contexto social, seguido de Observación del Proceso Escolar, luego está la seriación de la asignatura de Observación y Práctica Docente I, II, III y IV, que inicia en tercero y termina en sexto semestre. 
Es en las asignaturas de Observación y Práctica Docente donde el estudiante se acerca a la realidad educativa de los centros escolares. Así entonces, se establece que en Observación y Práctica Docente I, el estudiante realice dos Jornadas de Observación y Práctica Docente (JOPD) con una duración de una semana cada una, y en dos de los cinco días de la semana aplican la propuesta de español. Las cuáles,

En virtud del tiempo que demandan la preparación y aplicación de las observaciones y las prácticas, y por la complejidad de las mismas, es necesario recordar que los docentes del tercer semestre deben involucrarse en la planificación, la asesoría y el seguimiento de las mismas, así como en las actividades de los estudiantes (Programa de estudio, 2006, p. 15).

La asignatura de Observación y Práctica Docente II, también establece dos periodos de JOPD con duración de una semana cada una, de los cuales se ocupan dos días para acompañar/observar al maestro de educación especial y en los días restantes, aplican situaciones didácticas de español y matemáticas. El plan de estudio prescribe que,

Es necesario que el colegiado de cuarto semestre: a) participe en la organización de las jornadas de observación y práctica; b) asesore a los futuros docentes cuando éstos diseñen su guía de observación, sus situaciones didácticas de matemáticas, su propuesta de actividades de español, y su plan general de trabajo; c) supervise, con criterios comunes decididos en el colegio y con base en la temática que se estudia en cada curso de cuarto semestre, la realización de la jornada en las escuelas en que están distribuidos los estudiantes. Es indispensable que este trabajo se caracterice por la tolerancia, el diálogo, el compromiso y los acuerdos de consenso (Programa de estudio, 2006, pp. 13-14).

En quinto y sexto semestre el estudiante realiza las JOPD en el CAM, las cuales se dividen en dos periodos de práctica. El primero se realiza en una semana, dedicado a la observación y realización de ayudantía. El segundo, en dos semanas, exclusivas a la intervención psicopedagógica.

Para quinto semestre, se prescribe que,

El curso incluye actividades de observación y práctica preferentemente en servicios escolarizados de educación especial (CAM), en dos niveles educativos: preescolar y primaria; con el propósito de que los estudiantes diseñen y apliquen situaciones didácticas del currículo de educación básica. Con esto se pretende que los estudiantes se relacionen con los niños y los adolescentes que asisten al CAM, con los padres de familia y establezcan comunicación con los distintos profesionales que participan en este ámbito educativo (Programa de estudio, 2006, p. 2).

Respecto a los periodos de JOPD se plantea que,

La primera es de una semana, en la cual los estudiantes durante los tres primeros días observarán la dinámica del CAM y del aula que se les asigne, los dos días restantes continuarán con la observación, pero además apoyarán al maestro del grupo en la realización de actividades que este último les indique.

El conocimiento que obtenga de la primera jornada será esencial para el diseño y la planeación de la segunda, la cual tiene una duración de dos semanas continuas y deberá realizarse 
en el mismo grupo visitado para aprovechar el conocimiento obtenido en la primera visita; para esta segunda jornada los estudiantes observarán dos días para analizar y reflexionar sobre el diseño de situaciones didácticas que elaboraron para el grupo al que asistieron la primera jornada, con el propósito de que tal diseño pueda ser modificado o ajustado según las condiciones y características de la realidad educativa del CAM; los siguientes días aplicarán por lo menos dos situaciones didácticas diarias, además, el estudiante compartirá el espacio con el maestro del grupo, es decir, esta jornada no implica estar como responsable del grupo todo el tiempo, sino que habrá actividades que realice el maestro de grupo y apoyado por el normalista y otras que son su responsabilidad pero puede contar con el apoyo del maestro, de tal forma que las negociaciones y acuerdos que tenga con este último durante la primera jornada serán determinantes para el éxito de la segunda (Programa de estudio, 2006, p. 2).

En el sexto semestre, el estudiante llega a la última parte de las actividades de acercamiento a la práctica escolar. Continúa realizando las JOPD en el CAM donde las realizó en el quinto semestre. El periodo de las Jornadas se divide en dos.

Son dos jornadas: la primera es de una semana. En ella los estudiantes durante los tres primeros días realizarán actividades de observación y ayudantía, los dos días restantes continuarán observando, pero también aplicarán situaciones didácticas que previamente acordarán con el maestro del grupo. Esto implica que deberán planear y organizar todo lo necesario durante la misma semana.

El conocimiento que obtenga de la primera jornada será esencial para el diseño y planeación de la segunda, la cual tiene una duración de dos semanas continuas y debe realizarse en el mismo grupo visitado para aprovechar el conocimiento obtenido en la primera visita. Para esta segunda jornada los estudiantes observarán dos días para analizar y reflexionar sobre el diseño de la propuesta curricular adaptada que elaboraron para el grupo al que asistieron la primera jornada, con el propósito que pueda ser modificado o ajustado según las condiciones y características de la realidad educativa del CAM; los siguientes días aplicarán la propuesta y serán responsables del grupo todo el horario. Los estudiantes deben continuar acordando con el maestro titular durante las dos jornadas para garantizar que se esté brindando una atención educativa adecuada a los alumnos (Programa de estudio, 2006, pp. 8-9).

Una vez planteado el desarrollo de las JOPD para el estudiante de la licenciatura en Educación Especial, emerge la pregunta, ¿cómo asumen la formación inicial docente los estudiantes de la licenciatura educación especial en la Usaer y CAM donde realizan los periodos de práctica? El objetivo que atiende esta investigación es, analizar la asunción de la formación inicial docente de los estudiantes de la licenciatura en Educación Especial en la Usaer y CAM donde realizan las Actividades de Acercamiento a la Práctica Escolar.

La perspectiva teórica desde donde se analiza la formación inicial docente en el estudiante de la licenciatura en educación especial, es desde el planteamiento del saber docente. Dado que es el saber con el que se atiende la vida cotidiana (Heller, 2004). Pues en la vida cotidiana se encuentra lo común a todos, donde se establecen códigos que se legitiman, que incluso el incomodarse por algunas acciones es normal. La cotidianidad es parte del enfrentamiento en el diario vivir, de las ideas sometidas hacia el otro; las relaciones sociales del aula pueden ser transformadas a partir de reconocer la cotidianidad enajenada, esto es, del sometimiento del docente a ideas de otro (Becerril, 2005). 
En el saber docente, la situación que se enfrenta radica en la cotidianidad escolar, Mercado (2010) al respecto plantea que los profesores siempre saben cómo atender la cotidianidad escolar. Pues reproducen y construyen nuevas maneras de vivir en la escuela. Es por eso, que el recreo escolar, las clases, las horas de deporte, la interrelación con los padres de familia, se hacen familiares, se genera intimidad al interior del centro escolar, entre los actores que la hacen. En sí, toda la vivencia que se genera va configurando la vida escolar.

El docente en formación, inmerso en la socialización de la escuela, adquiere saberes formales que se constituyen por competencias básicas y profesionales pertinentes para su actividad. Pelletier y Morales-Perlaza (citado por Cantón \& Tardiff, 2018) refieren que durante las actividades profesionales el saber formal se transforma por las necesidades de adaptación y sobrevivencia del contexto laboral. El sentido de los saberes en el docente conlleva a una conformación plural y de complejidad sociológica de la identidad. El saber es estructural y representa poder.

Tardiff (2004) enuncia que los saberes docentes integran diferentes saberes de diversas fuentes, los cuales se constituyen en saberes disciplinarios, curriculares, profesionales (incluidos los de las ciencias de la educación y pedagogía) y experienciales. Estos saberes se pueden adquirir, desarrollar y contrastar en las actividades escolarizadas y en el acercamiento a la práctica escolar, es decir, durante la jornada pedagógica.

La formación inicial es un proceso pedagógico fundamental para la conformación de saberes docentes. Todo saber implica un proceso de enseñanza y aprendizaje; estos procesos resultan complejos cuando hay una mayor sistematización y formalización de las ciencias y los saberes. Los saberes suelen ser integrados a procesos de formación institucionalizados que son coordinados por los agentes educativos (Tardif, 2004).

La formación inicial docente no únicamente contempla el saber, sino que requiere de un saber hacer, esto es, que los docentes en formación puedan diseñar y llevar a la práctica propuestas de enseñanza en la que se labore planificaciones con la trasposición de lo aprendido en el aula. Marcelo y Vaillant (2009) señalan que los conocimientos adquiridos en la formación inicial docente aunados a la experiencia de la práctica actualmente no son suficientes, sino que precisan del desarrollo profesional continuo y permanente, esto por los cambios vertiginosos de las sociedades.

En el proceso de formación, el docente en formación se encuentra en implicadas experiencias y saberes pedagógicos, para Vergaras (2015) la formación considera el repensar el acto pedagógico y ver el interior de la escuela. Esto es, que se piense diferente acerca de las situaciones que se reconstruyen en la praxis del docente y analizar los relatos de los protagonistas en el proceso educativo. Además, los escenarios representan la oportunidad de repensar la práctica docente como una forma de reescribir la historia pedagógica para la comprensión y transformación.

La escuela considerada como escenario de operaciones didácticas, es un espacio de operatividad de procesos didácticos, el docente es un planificador. Es en la escuela donde se instituye un modelo pedagógico, de la misma forma es el lugar donde se dan los discursos didácticos, y aquí el docente toma un papel importante como traductor y filtrador de la enseñanza, esto contempla que sea un gestor real del desarrollo de la actividad del aula (Zabalza, 1987). 
La escuela representa un espacio de interacciones, en la que se reconstruyen los saberes, aquellos que se articulan a la teoría, y que, en el sentido propio de la práctica, el docente en formación toma aquello que forja su experiencia significativa. Después de todo, el docente es un agente social, que involucra a todos los que participan de la comunidad escolar, como tal, la escuela es pensada no sólo en el terreno de lo ideal e individual sino de lo fáctico y colectivo, Zabalza (1987) considera que,

la escuela en cuanto comunidad de gentes permite la gestación de unas programaciones plurales y consensuadas, que superan el marco estrecho de un aula y se enfrentan con un contexto más amplio de partida; que superan lo individual para centrarse en un nivel intermedio de lo colectivo (p.48).

Así, el docente se sitúa en entre su individualidad y colectividad de la escuela, pero su práctica adquiere importancia por la estructuración de significados que se establecen en la interacción con el otro. El docente se encuentra inmerso ante experiencias y vivencias, que van construyendo los saberes en lo cotidiano del aula.

El docente en formación inmerso en las prácticas pedagógicas de los contextos escolares aprende, reconoce y valora lo que ahí se vive. Charlot (2008), al respecto señala que,

El individuo formado es aquel que, a través de sus prácticas, es capaz de movilizar los medios y las competencias necesarias (a las suyas, pero también eventualmente a las de otros) para alcanzar un fin determinado en una situación dada. La práctica es direccionada: lo que da pertinencia es la relación entre medio y fin. La práctica es contextualizada: debe poder controlar la variación: no solo aquella previsible, normalizada, sino la minivariación, como desvío de la norma, como imprevisto, como expresión de la inestabilidad inherente e irreductible de cualquier situación (p. 92).

Así entonces, el saber que se construye en la práctica está en función de atender la cotidianidad. En el caso del saber docente, es con ese saber de práctica intencional establecido en el plan de estudio, que se forma el razonamiento, en la intención de comprender y entender al actor que hace la escuela: estudiante, profesor, entre otros. El plan de estudio, es entonces el representante de la razón institucional, el cual asumen los profesores como el modelo que orienta la práctica educativa y desde el cual configuran su hacer para la atención de los estudiantes (Zacarías \& Luna, 2018).

El saber ser docente se adquiere de la profesión docente, que si bien parte de ese saber vinculado con la racionalidad del plan de estudio, el hacerse docente tiene que ver en la manera en que se elabora esa presencia, en donde se tienen que pensar los modos en que se establecen las relaciones en el centro escolar (Contreras, 2010). Es reflexionarse en el saber, "en el modo propio de vivir las situaciones educativas, aquellas que nos permiten estar de verdad" (Contreras, 2010, p. 65).

El contexto de la realidad educativa, es pues el cultivo del saber de las certezas con la que se atiende la cotidianidad. Desde esas certezas se da al estudiante la validez del desempeño escolar, pues,

sabemos que una gran parte de toda esta forma de mirar nuestro trabajo, afrontar sus dificultades y proporcionar lo que está en nuestra mano y acompañar la formación de nuestros 
estudiantes, la hemos ido cultivando como nuestro personal bagaje profesional, conformando así un saber y un modo de saber que no se representa con los patrones de las materias, ni con las epistemologías en uso (Contreras, 2013, p. 127).

El saber docente entonces, si bien encierra el planteamiento del sistema educativo a través del plan de estudio, se fortalece con la cotidianidad del centro escolar. La intención por tanto de ese saber, sitúa un tipo de enseñanza para la continuidad de la cultura y de la formación ciudadana de las personas que la hacen.

En el discurso de lo cotidiano, los saberes disciplinares toman relevancia en la idealización de la formación inicial docente, pero dichos saberes, en cierta forma, son probados en lo escolar, aquello que pueda sustentar la práctica docente es con lo que el docente trabaja. La práctica docente mantiene un entramado de la vida teórica y lo que obtiene de la formación inicial docente y de la vida práctica, aquello que somete en experiencias de la vida en el aula.

\section{METODOLOGÍA}

La investigación se diseñó a partir del enfoque cualitativo Van-Manen (2003) \& Flick (2014), emplea el uso del método de análisis de contenido (Navarro \& Díaz, 2009) para la indagación de cómo se configura la formación inicial docente el estudiante de la licenciatura en educación especial a partir de la información que registra en los diarios de trabajo que realiza al terminar la Jornada de práctica docente de un día.

En este sentido, se solicitó a estudiantes que cursan el tercer y quinto semestre de la licenciatura en educación especial de la escuela normal de licenciatura en Educación Especial de la Escuela Normal de Licenciatura en Educación Primaria, en Tapachula, Chiapas; que permitieran leer sus diarios de trabajo que elaboran en las Jornadas de Observación y Práctica Docente. Se revisaron 44 diarios de trabajo, de un total de 44 estudiantes inscritos en ambos semestres.

A partir del corpus de datos, se seleccionó a cuatro diarios de trabajo (dos de tercer semestre y dos de quinto semestre), donde estuvieran contenidas las ideas que hacían común la sistematización de un día de JOPD. El proceso implicó la lectura atenta de qué es aquello que toman como relevante, qué reflexionan de la intervención realizada y cuáles son las dudas que se generan durante la jornada pedagógica.

Para el análisis de la información obtenida se estableció el criterio de comparación de los textos escritos de cuatro fuentes en diferentes situaciones (Bardin, 1986), de donde los datos cifrados condujeron a la estructuración de las subjetividades enfocadas a vivencias de la práctica docente, los cuales representa el discurso del alumno.

En relación al análisis de los textos escritos (diarios de trabajo) se elaboraron 3 preguntas para atender el contenido: ¿Qué descripciones deriva de la jornada de práctica docente? ¿Qué reflexiones hace de las impresiones? y ¿qué dudas le emergen de la cotidianidad vivida en un día en el centro escolar? 


\section{RESULTADOS}

Una vez analizado el corpus del contenido textual de los diarios, se encontró que los estudiantes describen lo que sucede en el aula: las relaciones sociales de los actores y el trabajo pedagógico.

La maestra inició cantando una canción, luego preguntando y mencionando el nombre del alumno. De acuerdo a las características que presenta el alumno, noté que presenta Trastorno del Espectro Autista. El alumno hacia movimientos involuntarios y se metía la mano en la boca para morderse los dedos, por eso mismo su tía, quien estaba a su lado, lo tomaba de un brazo y le quitaba a cada ratos la mano de la boca, o lo sostenía muy fuerte para que el alumno no se auto golpeara, por su parte, la maestra intentaba enseñarle y también le ayudaba a quitarse la mano o la playera que se echaba a la boca, pero en un momento determinado, el estudiante se veía desesperado y comenzó a golpear la mesa con los pies y las manos (A14-5S).

Constaban en el aula cinco alumnos con diferentes discapacidades, inicié la clase con una retroalimentación del tema del plato del bien comer, pedí la participación de los alumnos para orientar al niño ciego, después de una media hora de estar explicando, les dejé una actividad que consistía en que los alumnos identificaran las verduras y frutas que consumiera en casa, para el niño ciego le llevé material con relieve para la identificación de sus alimentos que consume (A6-5S).

Las maestras tanto de Usaer y regular me estuvieron apoyando en el control del grupo y aconsejando, por ejemplo, en que no debo de dejar de hablar y de llamar su atención, aunque se esté realizando la actividad porque si no, ellos pierden la atención de lo que están haciendo y se distraen con sus compañeros o con otras cosas, y si uno se distrae, los demás empiezan a perder interés en la actividad. Considero que esa actividad no tuvo el resultado esperado, tanto por el material que se usó y por la forma de realizar la actividad (A11-3S).

En este día al iniciar la jornada en la escuela Fray Matías de Córdova, al iniciar la jornada, la maestra pasó lista y todos los niños asistieron, en cuanto terminó el niño que siempre se la pasa haciendo escandalo estaba callado y otro niño dijo "Kevin anima el salón esta todo aburrido", todos se empezaron a reír. La maestra los regañó y el niño habló, "estoy enfermo de la garganta" (voz ronca). La maestra me dijo que saldría un rato y que interviniera porque después se irían a música. Inicié con la tarea, casi nadie la entrego, les dejé una actividad, todos participaban y como era de esperarse, como todo el grupo hacía mucho escándalo (A20-3S).

En cuanto a las descripciones que emergen de un día de JOPD, los estudiantes se centran en aspectos de asombro, pero se involucran, además, saberes disciplinarios al hacer alusión de una necesidad educativa especial y los elementos pedagógicos como materiales didácticos pertinentes para generar aprendizaje. Aunque se puede comprender saberes experienciales implicados en la cotidianidad del contexto escolar.

En relación a cómo asume el estudiante el proceso formativo en educación especial a partir del acercamiento a la práctica educativa, se destaca lo siguiente: 
El maestro no solo es el que imparte la clase, sino también es consejero, el que se encarga de los problemas que se presenten con sus alumnos sin tomar partido por nadie (A20-3S).

Este último día, considero que me sirvió para identificar ciertas cosas que tengo que mejorar al trabajar con todo el grupo y de manera individual. También para concientizarme más de que estoy trabajando con alumnos de preescolar y se necesitan de muchas actividades que llamen su atención e interés, ya que jugar es lo que les gusta hacer. Aunque trabajar con todo el grupo no sea una de las actividades que realiza en sí una maestra de Educación Especial, pienso que ayuda mucho para realizar adecuaciones a las actividades diarias que se realizan con todo el grupo (A11-3S).

Realmente las experiencias te marcan en gran magnitud, no me había enfrentado a un trabajo en CAM, es realmente sorprendente que los docentes puedan trabajar con la gran cantidad de alumnos que cuenta cada salón. Esta primera jornada de prácticas me benefició para conocer más al grupo, sus gustos, cómo trabajan, aunque sé que me falta mucho, así mismo al no tener la valoración de cada alumno no se puede realizar un trabajo adecuado (A14-5S).

En general, terminé demasiado agotada, incluso más que cuando estuve a cargo del grupo en el aula, considero que éste agotamiento se debe a que no es lo mismo realizar una actividad al aire libre como en el salón, ya que el hecho de estar a la intemperie con los alumnos implica que ellos se desplacen en terrenos más extensos y por ende yo tengo que desplazarme tanto como ellos lo hagan para estar al pendiente de ellos, y no solamente de mi grupo, ya que también terminé cuidando a alumnos de los demás grupos y eso fue satisfactorio para mí (A6-5S).

El estudiante analiza su actividad realizada durante la jornada, relaciona sus experiencias al saber docente, lo que conlleva a reflexionar su práctica educativa, y expresa admiración por la labor del docente de educación especial, además de reconocer que hay actividades que no son propias; hay elementos coincidentes como sorpresa y agotamiento en el advenimiento de la práctica docente.

Las dudas, impresiones e incertidumbres con las que cierran los diarios de trabajo los estudiantes, no precisamente refieren a la vivencia del día, sino, que se tornan problematizadoras del quehacer docente.

Mis impresiones del día de hoy fueron acerca de dudas, incertidumbres y nervios al buscar la implementación de actividades con el alumno que presenta TEA, de igual manera me causaron dudas, como las siguientes; ¿Cómo trabaja la profesora cuando asisten en un día todos los alumnos de su salón?, ¿Por qué, si le dan medicamentos al alumno, no se observan cambios en su conducta? ¿Por qué si el alumno estaba enfermo lo llevaron al CAM así? ¿Con qué alumno se le es más complicado trabajar la maestra? (A14-5S).

Cabe recalcar que las actividades estructuradas de hoy, no estuvieron contempladas en mi planeación como tal. Con base a este día me impresionó que los alumnos tiendan a prestar más atención cuando le enseñas algo novedoso, y aunque la actividad sea muy dinámica si ya la practicaron con anterioridad no les generará interés (A6-5S). 
Por consiguiente, Isaac apertura la actividad lanzando el cubo, pero con frecuencia emite comentarios referentes a que no quiere hacer la actividad y a que se encuentra cansado. Esto me genera conflicto, ya que no había pasado mucho tiempo desde que se ofreció para dar inicio, pero me decido a animarlo. Durante su participación pude percatarme de que Santiago con frecuencia intentaba ayudarlo, interpretando el verso según el tono de voz que le correspondía a su compañero, lo que en primer lugar consideré como algo factible para que éste viera el ejemplo, pero después decidí comentarle a Santiago que dejará de hacerlo, pues debía aprender a respetar turnos, favoreciendo a su vez la autonomía de Isaac para resolver por sí solo la situación a la que se encontraba expuesto... aunque me surgen dudas relacionadas con el porqué Isaac siempre se muestra con esa actitud durante las clases, pues a esto se suma que no realiza las actividades en el aula, ni en casa (A11-3S).

En estos cuatro días me he podido dar cuenta que tuve una rutina en el salón de clases: llegar y ayudar a la maestra en lo que necesite, sacar la silla de rueda y esperar a Martha, llevarla al salón de clases y esperar a que entre la maestra. Tratar de que Martha realice las actividades, cuidar que no se lastime, que no se esté mordiendo, acomodarla cada vez que se resbala de la silla, darle de comer, estar con ella en receso, también ayudar a los alumnos si necesitan algo, vigilarlos cuando la maestra sale del salón, buscar material que la maestra no puede encontrar, etcétera. Me gustaría que la semana de enero pueda apoyar de manera más significativa a los alumnos con Necesidades Educativas, conociendo con anticipación los temas que van a ver y adecuarlos principalmente a Martha. No sé con exactitud si esta es la labor que realizan las maestras de Educación Especial, pero me agradaría realizar un aporte específico (A20-3S).

El estudiante en formación en el desarrollo de su práctica presenta incertidumbre ante aquello que desconoce o de lo cual tiene nociones, pero aún no ha enfrentado, esto indica que la formación inicial docente estructura experiencias teóricas-conceptuales, pero no un proceder sobre lo práctico, sobre aquello que sucede en las aulas. Esto conlleva a pensar en el mundo de vida idealizado que se tiene en las escuelas normales, pero que en el terreno de las vivencias adquiere predicamento lo que de solución a las dificultades de la enseñanza y aprendizaje.

Por otro lado, el estudiante cuestiona la práctica docente ante las diferencias encontradas en el aula, cómo aborda el docente la enseñanza y qué tipo de Necesidad Educativa Especial le presenta un mayor reto. De igual forma, el currículo oculto se presenta en acciones discrepantes a la planeación docente. Pues el estudiante da cuenta de que hay situaciones no esperadas fuera de toda planificación, y otras que se encuentran prescritas en prácticas habituales que conforman una rutinización de la práctica docente.

\section{DISCUSIÓN}

Si educar en estos tiempos es reconocer la diferencia del otro para estar con él, entonces, la formación de la docencia, trasciende la prescripción de un plan de estudio en torno a la enseñanza del ideal ciudadano a formar. Pues, a partir de la información que se analizó en los diarios de clase de los estudiantes, se encontraron tres situaciones: reconocer qué se vive en la cotidianidad escolar, cómo enseñan los contenidos escolares, y qué problematizaciones emergen de la realidad educativa observada. 
labras claves que se tienen que emplear para que los estudiantes presten la atención: callar al grupo, darles imperativos, ser prescriptivo y restrictivo. Todo lo anterior, es reproducido cuando se les pide que se queden a cargo del grupo de alumnos, o bien, cuando la ayudantía de la práctica docente, se centra en un estudiante.

El saber docente con el que el profesor titular -donde el estudiante realiza el acercamiento a la práctica escolar-, atiende la cotidianidad escolar, es el que registra el estudiante en los diarios. Con ese saber que observa va configurando su formación inicial docente, pues reconoce no solo las estrategias didácticas para trabajar los contenidos escolares del plan de estudio, sino, para la relación de convivencia con el estudiante.

El estudiante refiere que el profesor, asume su práctica docente como una expresión de saberes prácticos, entonces lo aplicable, lo que tiene un sentido práctico, lo que funciona es lo que conforma las vivencias del aula, lo que fortalece la interacción didáctica entre el docente y alumno, en donde se atribuye procesos no sólo de enseñanza y aprendizaje sino sociales y culturales. Las diferencias que encuentra el estudiante en formación en sus vivencias escolares durante su observación y práctica dan cuenta de que el estudiante establece la formación inicial docente como una formación teórica y lo que ocurre en la cotidianidad como una formación práctica. Así, el dilema de lo teórico y práctico de la docencia evidencia una fragilidad de la función docente porque los eventos del aula no son los mismos a pesar de la rutinización docente.

El estudiante cuestiona la práctica docente que enfrenta en el aula en su posición de observador, allí da cuenta de que el docente en su práctica tiene una determinada forma de enseñanza y que sin duda existen retos que dificultan su función como docente; el estudiante que cuestiona la práctica docente, infiere que la formación docente y sus saberes disciplinarios dista de la realidad escolar. El estudiante se encuentra ante un currículo oculto, los actos de la cotidianidad, la cultura escolar son situaciones que no espera, que están fuera de todo acto de planificación. No obstante, hay situaciones que se vuelven parte de la cotidianidad, que conforman una forma de rutina en el docente, la rutinización como una forma de creencias en el docente, asegura un tipo de comportamiento en el docente, es decir, de actuar conforme lo que le sirve en el aula.

Así entonces, la formación inicial docente de estos estudiantes, se problematiza al dar cuenta de lo agotador que puede ser un día de jornada escolar, de la innovación constante que tienen que hacer los profesores para captar el interés de todos los estudiantes, de lo que tiene que conocer teóricamente el profesor para enseñar el contenido escolar de acuerdo a la necesidad educativa que presente el estudiante. Más, qué hay en esa decisión de los padres de familia al dejar a su hijo con situaciones de salud inestables en la escuela a cargo del profesor.

Los saberes experienciales adquiridos durante las jornadas pedagógicas, se encuentran fincados en el trabajo cotidiano que se realiza al igual que el conocimiento del contexto escolar, la reflexión que el estudiante de educación especial hace de su práctica implican los saberes disciplinares, por ello, la práctica pedagógica no está constituida por un solo tipo de saber, sino de saberes obtenidos de diversas fuentes (Tardif, 2004). 
Así entonces, cuando el estudiante refiere que los profesores en servicio -sea de Usaer, de CAM o aula regular-, respecto a la atención que se da en el momento a los estudiantes que presentan necesidades educativas, deja ver que la función docente está en función de la prescripción que el Sistema Educativo Mexicano establece para su atención. A la vez, dan cuenta de las inmediateces que suceden en el aula, por ejemplo, la angustia que le produce a la estudiante de educación especial, del por qué llegan estudiantes enfermos al aula, tener la inseguridad de que, si lo que hace la profesora titular del grupo, es lo que tiene que hacer una profesora de educación especial.

Se destaca en la narración que hacen las estudiantes en el diario, que es en el contexto de la realidad educativa donde se fortalece la formación inicial docente, pues les permite entender que la vida cotidiana escolar fortalece el carácter de ser profesor para la educación especial. Así, por ejemplo, cuando se hace referencia que se ha quedado a cargo del total del grupo en el que son asignados, como si fueran los profesores de grupo, el estudiante asume de que no solo es el contenido académico el que tiene que trabajar, sino que, es toda la emoción que en ese momento se refleje. Incluso cuando se hace referencia a que se tiene que fortalecer las habilidades para el control de grupo, es un sentir que va identificando necesario para poder impartir clase. Sentir que va fraguando en estos acercamientos a las actividades de la práctica escolar, tal como plantea el plan de estudio en el perfil de egreso.

Las experiencias que se obtienen de la práctica docente, enriquecen la conformación de ser docente, pues este no únicamente se establece de la formación inicial docente, sino que se van agregando situaciones significativas que crean en el estudiante un estilo de enseñanza, así como conocimiento prácticos que infieren su práctica, esto porque los saberes que se expresan en el aula son saberes propios, saberes con base en la experiencia, entonces, en la cotidianidad escolar dichas experiencias sustentan su saber docente, a ello, se tendría que cuestionar que la reflexión de la práctica docente es una actitud necesaria, además de orientar su saber-hacer pedagógico, otorga significado a su proceso de ser docente; situando el tema de los saberes, se tiene entonces, que los saberes disciplinares son trascendentes a la función docente, sin embargo, los saberes de la experiencia de la práctica repercuten en la subjetividad docente, creando un sentido de identidad y pertenencia.

Si bien en un fragmento del diario se menciona algunas necesidades educativas que encuentran en el aula y se reconocen las actividades que realizan las profesoras titulares -en este caso del grupo de CAM-, esta identificación de necesidades resulta de vital importancia, porque de acuerdo al plan de estudio para la formación del licenciado en educación especial (2004), el estudiante se forma con conocimientos teóricos de psicología, medicina y pedagogía. Resulta entonces, que la singularidad que fortalece al tener los acercamientos a los contextos de la práctica escolar, pone en dinámica la teoría que aprende de las tres disciplinas, pues como se lee en los diarios, no solo da cuenta de qué cantos ocupa la maestra, sino que también nombra como a aprendido en los cursos que lleva en la licenciatura la discapacidad que puede presentar un estudiante con necesidades educativas.

El saber que el estudiante adquiere en el acercamiento al contexto de la práctica escolar que tiene en cada JOPD se centra en tres aspectos: ver, escuchar y reproducir. Ve lo que hacen los profesores titulares con los estudiantes -le cantan canciones, moderan la conducta agresiva del estudiante, el profesor es consejero-, así mismo, reconoce cuáles son las pa- 
El saber que construyen los estudiantes a partir de las JOPD les da elementos para configurar la cotidianidad escolar. Aprenden la manera en que se trata al alumno, las rutinas escolares, y las estrategias que hay que usar ante situaciones previsibles o no para la atención de los estudiantes (Contreras, 2010, 2013).

\section{CONCLUSIONES}

$\mathrm{Al}$ analizar la asunción de la formación inicial docente en los estudiantes de la licenciatura educación especial en la Usaer y CAM a partir de los diarios de clase que elaboran en las JOPD -las actividades de acercamiento a la práctica escolar-, se encuentra que la impresión de la vida cotidiana escolar es donde centran su atención al redactar las vivencias de un día: ver, escuchar, reproducir, la asumen como la cotidianidad que no viven pero, que auguran que esas vivencias tendrán cuando realicen las prácticas intensivas en condiciones reales de trabajo, y cuando sean los profesores titulares para atender la Usaer o CAM. Así entonces, la formación inicial docente para la educación especial que asumen los estudiantes se entreteje entre el planteamiento de la prescripción del currículo escolar, y el saber con el que atienden la cotidianidad escolar los profesores. Por tal, betas de indagación, 1) el estudiante que realiza prácticas intensiva en condiciones reales de trabajo, qué saberes docentes posee al terminar la formación como licenciado en educación especial, 2) al interior de la escuela normal, qué saber docente comunica el formador, 3) cómo convergen en la formación inicial docente para la educación especial, el conocimiento teórico que establece el plan de estudio licenciatura, y la experiencia que ha tenido el profesor-formador en torno a la educación especial.

La cotidianidad del contexto implica saberes basados en la experiencia, pero, además, los saberes relativos a la formación profesional, el conocimiento es un cuerpo de interpretación teórica de la realidad, el estudiante al vivir las situaciones educativas de su práctica manifiesta el uso o rechazo de los saberes y esto mismo indica la preponderancia de la formación inicial para las actividades que realizará.

Lo anterior denota la exterioridad de la práctica docente, porque, aunque en la formación inicial los saberes científicos y pedagógicos constituyen la identidad de formación docente no determina que los contenidos aprendidos en el aula sean de utilidad en sus actividades. Es por ello que, en el diario de trabajo, se aprecia el discurso docente, el cual se nutre por las vivencias obtenidas, y estos discursos reflejan la conformación docente.

\section{REFERENCIAS}

Bardin, L. (1986). El análisis de contenido. Akal.

Becerril Calderón, S. (2005). Comprender la práctica docente. Categorías para una interpretación científica. Plaza y Valdés, S.A.

Cantón M., I. y Tardif M. (2018). Identidad profesional docente. NARCEA.

Charlot, B. (2008). La relación con el saber, formación de maestros y profesores, educación y globalización. Cuestiones para la educación de hoy. TRILCE. 
Contreras Domingo, J. (2010). Ser y saber en la formación didáctica del profesorado: una visión personal. Revista Interuniversitaria de Formación del Profesorado, 24(2), 61-81.

Contreras Domingo, J. (2013). El saber de la experiencia en la formación inicial del profesorado. Revista Interuniversitaria de Formación del Profesorado, 27(3), 125136.

Flick, U. (2014). La gestión de la calidad en investigación cualitativa. Morata.

Heller, A. (1977). Sociología de la vida cotidiana. Península.

Marcelo, C. \& Vaillant, D. (2009) Desarrollo profesional docente ¿Cómo se aprende a enseñar? NARCEA.

Mercado, M. R. (2002). Los saberes docentes como construcción social: La enseñanza centrada en los niños. Fondo de Cultura Económica.

Navarro, P., \& Díaz, C. (2009). Análisis de contenido. En J. M. Delgado \& J. Gutiérrez (Coords). Métodos y técnicas cualitativas de investigación en ciencias sociales (pp. 177-224). Síntesis.

Tardif, M. (2004). Los saberes del docente y su desarrollo profesional. Narcea.

Van-Manen, M. (2003). Investigación educativa y experiencia vivida. Ciencia humana para una pedagogía de la acción y la sensibilidad. Idea Books, S. A.

Vergaras, A. (2015). El saber pedagógico. Reconstrucción social de la praxis educativa. EAE.

Zabalza, A.M. (1987) Diseño y desarrollo curricular. NARCEA.

Zacarías, M. \& Luna, M. J. E. (2018). Saber docente para la atención del servicio de USAER en una región de Chiapas. Revista Praxis Investigativa ReggDIE,10(18), 79-90. 